\title{
Emergence and clonal dissemination of Salmonella enterica serovar Enteritidis causing salmonellosis in Mauritius
}

\author{
Mohammad I Issack ${ }^{1}$, Rene S. Hendriksen², Eija Hyytiä-Trees ${ }^{3}$, Christina A. Svendsen², Matthew \\ Mikoleit $^{3}$ \\ ${ }^{1}$ Central Health Laboratory, Victoria Hospital, Candos, Mauritius \\ ${ }^{2}$ WHO Collaborating Centre for antimicrobial resistance in foodborne pathogens and European Union Reference \\ Laboratory for antimicrobial resistance, National Food Institute, Technical University of Denmark, Kgs. Lyngby, \\ Denmark \\ ${ }^{3}$ Centers for Disease Control and Prevention; National Center for Emerging and Zoonotic Infectious Diseases; \\ Division of Foodborne, Waterborne, and Environmental Diseases; Enteric Diseases Laboratory Branch, Atlanta, \\ Georgia, United States
}

\begin{abstract}
Introduction: For decades, Salmonella enterica serovar Enteritidis has been among the most prevalent serovars reported worldwide. However, it was rarely encountered in Mauritius until 2007; since then the number of non-typhoidal Salmonella serogroup O:9 (including serovar Enteritidis) increased. A study was conducted to investigate the genetic relatedness between $S$. Enteritidis isolates recovered in Mauritius from food and clinical specimens (stool, blood, and exudate).

Methodology: Forty-seven isolates of $S$. Enteritidis obtained in 2009 from human stools, blood cultures and exudates, and from food specimens were characterized by antimicrobial susceptibility testing and Multiple-Locus Variable-number tandem repeat Analysis (MLVA). Results: With the exception of a single isolate which demonstrated intermediate susceptibility to streptomycin, all isolates were pansusceptible to the 14 antimicrobials tested. Thirty seven out of the 47 isolates $(78.7 \%)$ exhibited an indistinguishable MLVA profile which included isolates from ready-to-eat food products, chicken, and human clinical isolates from stool, blood and exudate.

Conclusions: The presence of highly related strains in both humans and raw chicken, and the failure to isolate the serovar from other foods, suggests that poultry is the main reservoir of $S$. Enteritidis in Mauritius and that the majority of human cases are associated with chicken consumption which originated from one major producer. Stool isolates were indistinguishable or closely related to blood and exudate isolates, indicating that, besides gastroenteritis, the same strain caused invasive infections. Control of $S$. Enteritidis by poultry breeders would lower the financial burden associated with morbidity in humans caused by this organism in Mauritius.
\end{abstract}

Key words: Salmonella Enteritidis; antimicrobial resistance; human infections; MLVA; Mauritius

J Infect Dev Ctries 2014; 8(4):454-460. doi:10.3855/jidc.3695

(Received 18 April 2013 - Accepted 22 November 2013)

Copyright (C) 2014 Issack et al. This is an open-access article distributed under the Creative Commons Attribution License, which permits unrestricted use, distribution, and reproduction in any medium, provided the original work is properly cited.

\section{Introduction}

Non-typhoidal salmonellae (NTS) are among the most commonly reported causes of bacterial foodborne illness worldwide [1-3]. In Mauritius, an upper middle income island nation of 1.25 million inhabitants in the southwest Indian Ocean, Salmonella enterica has been the bacterial pathogen most frequently isolated from stools of patients for the last 12 years [4,5] and Salmonella serovar Typhimurium ( $S$. Typhimurium) has been the most commonly encountered serotype; $45 \%$ of isolates serogrouped between 1998 and 2009 at the Mauritius Central Health Laboratory (CHL) were O:4, the serogroup of $S$. Typhiurium.

$S$. Enteritidis is consistently among the two most common serovars reported from clinical specimens worldwide [6]. In Mauritius, serogrouping is typically performed in lieu of full serotyping. With the exception of one blood isolate in 2006 of nontyphoidal Salmonella serogroup O:9, which could have been $S$. Enteritidis. Salmonella Enteritidis was not isolated from stool or blood samples collected between 1999 and 2006 in Mauritius. In 2007, nontyphoidal Salmonella serogroup O:9 was isolated from two specimens and subsequently, its prevalence increased tremendously in 2008 to become the leading serogroup among stools and blood isolates (CHL data).

Since 2008, non-typhoidal Salmonella serogroup O:9 has been isolated in several instances in pure growth from human soft tissue and internal organ 
abscesses at the CHL in Mauritius and a total of 33 cases of non-typhoidal Salmonella serogroup 0:9 bacteraemia have been reported in 2008 and 2009 as shown in Figure 1. Salmonella Enteritidis has also been isolated at the CHL since 2008 from several specimens of raw chicken and ready-to-eat food in Mauritius and has been the cause of many foodborne disease outbreaks notified to public health services. However, little is known about the local epidemiology of this serotype.

The objective of the present study was to investigate genetic relatedness among $S$. Enteritidis isolates recovered from food and clinical samples associated with gastroenteritis, bacteremia and other extraintestinal infections in patients from Mauritius. By identifying potential reservoirs of $S$. Enteritidis, this study was also intended to find biological and epidemiological evidence of the probable source of these infections and identify potential interventions aimed at reducing morbidity and mortality caused by $S$. Enteritidis in Mauritius.

\section{Methodology}

\section{Isolation of Salmonella from different sources}

The CHL processes all clinical samples sent for bacteriologic investigations from all government healthcare institutions in Mauritius, as well as samples from some private medical clinics. Additionally, the CHL also processes all food samples for microbiologic testing collected by Ministry of Health inspectors during routine inspections or as part of the investigation of reported cases of foodborne illness.

All stool specimens were cultured directly onto Xylose Lysine Deoxycholate (XLD) agar (Oxoid, Basingstoke, UK) and enriched in selenite enrichment broth (Oxoid, Basingstoke, UK) followed by subculture on XLD agar. Agar plates and broth were both incubated at $37^{\circ} \mathrm{C}$ for 18 hours. Blood cultures were processed manually by standard procedures [7]. Salmonella in raw and in ready-to-eat food specimens was detected by standard methods [8] and identified using biochemical tests [9]. Serogrouping was performed by agglutination with monovalent $\mathrm{O}$ antisera (Mast, Bootle, United Kingdom; Remel, Dartford, United Kingdom).

Laboratory registers of stool specimens received in 2009 were reviewed for Salmonella-positive cases. Duplicate isolates from the same patient, and isolates from specimens sent for screening purposes were excluded. Additionally, specimen registers of blood and exudates cultured in 2009 were searched for Salmonella. Records of the microbiological testing of
Figure 1. Number of Salmonella serogroup 0:9 isolated from different sources at the Central Health Laboratory, Mauritius; 19982009

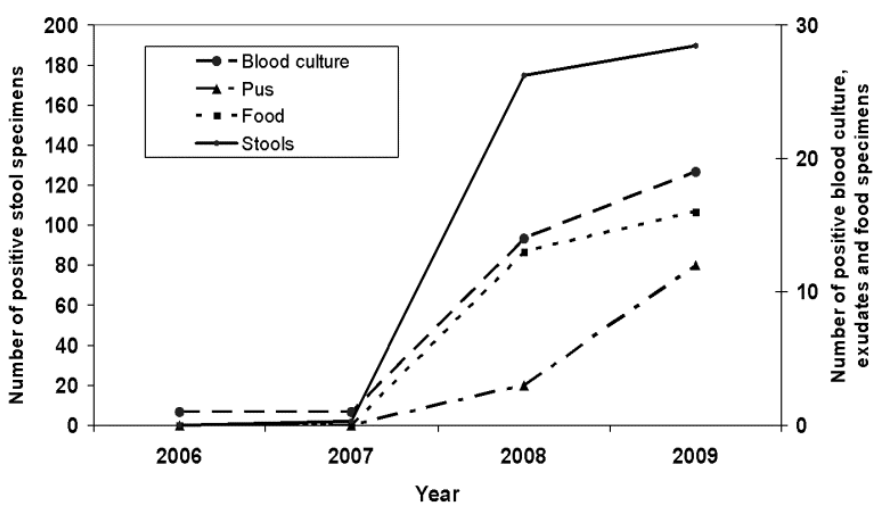

food specimens performed in 2009 at the CHL were also reviewed for foodstuffs positive for Salmonella. Records for 2006, 2007 and 2008 were also examined for trends in the isolation of Salmonella.

\section{Selection of isolates}

Forty-seven isolates of Salmonella serogroup O:9 isolated during 2009, all suspected to be $S$. Enteritidis were selected and sent to the National Institute of Health, Thailand for full serotyping, National Food Institute (DTU-FOOD), Denmark, for minimum inhibitory concentration (MIC) determination, and the Centers for Disease Control and Prevention (CDC), United States of America for Multiple-Locus Variablenumber tandem repeat Analysis (MLVA).

The isolates originated from food $(\mathrm{n}=16)$, blood culture $(n=9)$, abscesses $(n=4)$ and stool $(n=18)$ specimens (details in Table 1). At the CHL, all Salmonella isolates recovered from food are routinely saved; however clinical isolates are only saved if further studies are contemplated. In 2009, an increase in the burden of salmonellosis due to $S$. Enteritidis with a potential link to food was noted and as such, all clinical isolates from November and December 2009 were saved. Additionally, five blood culture isolates from January 2009 were available and were included to obtain information on possible temporal changes in strains causing infections. Isolates were selected based on availability and to ensure representation of food, stool, blood, and exudate isolates. Additionally, stool isolates were selected to ensure a mixture of both sporadic cases and outbreak cases. 
Table 1. Meta data of the 47 isolates of Salmonella serovar Enteritidis

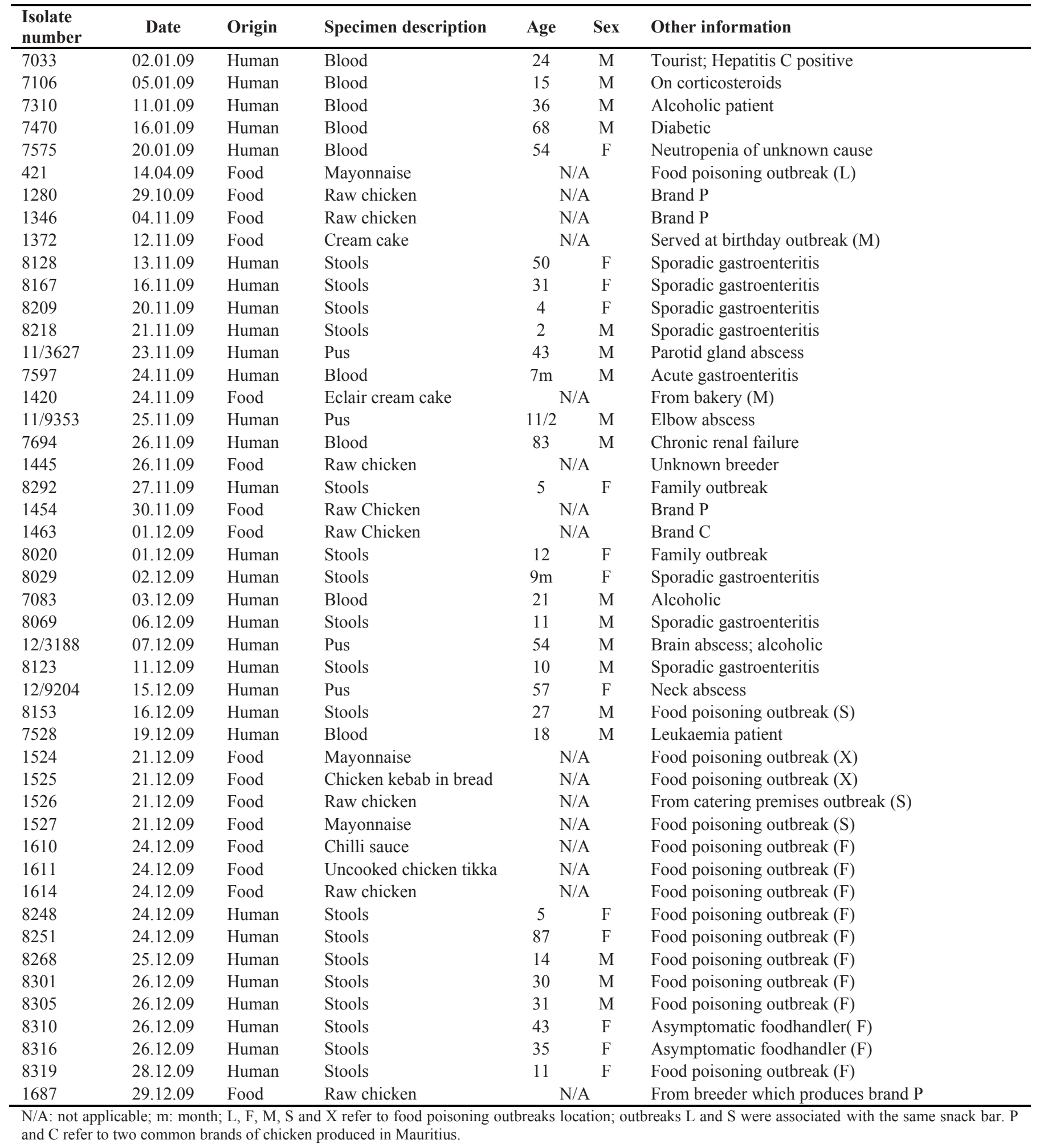




\section{Epidemiological data of selected isolates}

Epidemiological data on the isolates are summarized in Table 1. Stool isolates \#8248, \#8251, \#8268, \#8301, \#8305 and \#8319 were obtained from patients affected in a large outbreak that resulted in 700 persons seeking medical care and 105 requiring admission. It was linked to consumption of chicken tikka or kebab with home-made mayonnaise and chili sauce bought at a twice-weekly village market. Isolates \#8310 and \#8316 were recovered in the context of the outbreak investigation from stools of asymptomatic food handlers. Food isolates \#1610, \#1611 and \#1614 were obtained from samples collected at the premises where the chicken were marinated and the home-made mayonnaise and chilli sauce prepared. The caterer reported that the chicken used originated from the same major poultry breeder which markets the raw chicken from which isolate \#1687 was subsequently obtained. Moreover, isolates \#1280, \#1346 and \#1454 had previously been found in a brand of raw chicken produced by the same breeder. In contrast, isolate \#1463 was found in a brand of chicken produced by a different major poultry breeder and isolate \#1445 was obtained from chicken claimed to have been bought at a poultry shop which is supplied by an unspecified small breeder.

Food isolates \#1526 and \#1527 were obtained from the premises of a snack bar where chicken kebab that was associated with a small outbreak of salmonellosis was prepared; isolate \#8153 was from the stools of a patient who became ill in this outbreak. Isolate \#421 had previously been found in mayonnaise from the same snack bar following investigation of another small outbreak though no positive human specimen was obtained in that instance. Following an outbreak at a birthday party, food isolate \#1372 was found in one of the cream cakes that were served and subsequently isolate \#1420 was obtained from an éclair cream cake that was prepared in the bakery which supplied the cakes. Isolates \#8292 and \#8020 were obtained from two siblings who were admitted to hospital with food poisoning. Food isolates \#1524 and\#1525 were found in specimens collected at a food outlet following complaints by people who became ill after eating food sold there.

\section{Serotyping}

Isolates were serotyped using slide agglutination with hyperimmune sera (S \& A reagents lab, Ltd, Bangkok, Thailand) characterizing the $\mathrm{O}$ and $\mathrm{H}$ antigens. The serotypes were assigned according to the Kauffmann-White scheme [10]. At CDC, the serotype was confirmed by PCR amplification of the $S d f$ I gene specific for Salmonella serovar Enteritidis [11].

\section{Antimicrobial susceptibility testing}

Susceptibility to 17 different antimicrobial agents was performed by MIC determination at DTU-Food, Denmark using a commercially prepared, panel of dehydrated antimicrobials (Sensititre; TREK Diagnostic Systems Ltd., East Grinstead, England). [12]. Results were interpreted using EUCASTrecommended cutoff values accessed at http://www.eucast.org.

\section{Multiple-Locus Variable-number tandem repeat} Analysis

MLVA was performed by following the standardized PulseNet USA protocol for S. enterica serotype Enteritidis (Laboratory standard operating procedure for PulseNet MLVA of S. enterica serotype Enteritis - Beckman Coulter 8000 platform. Accessed at: www.pulsenetinternational.org). Comparisons were performed using Bionumerics software version 5.01 (Applied Maths, Sint-Martens-Latem, Belgium) using the categorical coefficient and unweighted pair group method with arithmetic mean (UPGMA) clustering. The profiles were compared against the PulseNet USA national MLVA database.

\section{Results}

\section{Isolation of Salmonella from different sources}

Laboratory registers of stool specimens received in 2009 revealed 336 NTS cases of which 190 (56.5\%) were Salmonella serogroup O:9. From 11,330 blood culture bottles received in 2009, 31 cases of proven NTS bacteraemia were identified of which $19(61.3 \%)$ belonged to Salmonella serogroup O:9. Moreover, NTS were isolated from exudates from soft tissue and internal organs from 15 patients in 2009 and in 12 $(80 \%)$ cases, the causative organisms was Salmonella serogroup O:9.

Records of the microbiological testing of 1,770 food specimens performed in 2009 showed 24 Salmonella-positive samples of which $16(67 \%)$ were due to Salmonella serogroup O:9.

Results showing trends in the number of Salmonella serogroup 0:9 from different sources are summarized in Figure 1.

\section{Serotyping and Antimicrobial resistance}

All isolates were identified as $S$. Enteritidis and were susceptible to ampicillin, apramycin, cefotaxime, ceftiofur, chloramphenicol, ciprofloxacin, florfenicol, 
gentamicin, nalidixic acid, neomycin, spectinomycin, sulphamethoxazole, tetracycline, and trimethoprim. One stool isolate (\#8305) exhibited intermediate susceptibility to streptomycin.

\section{Multiple-Locus Variable-number tandem repeat Analysis}

Thirty seven isolates (78.7\%) exhibited the MLVA allele profile 4-7-2-10-2-4-10. The remaining isolates exhibited four different profiles, all of which were highly related to the main profile, by differing at a single locus by 1 to 2 repeat units. All five profiles were rare in the United States MLVA database with prevalence ranging from $0.05 \%-0.16 \%$. The main profile was found in $16(89 \%)$ of 18 human stool isolates, six $(67 \%)$ of nine blood culture isolates, three $(75 \%)$ of four exudate isolates including an isolate recovered from a brain abscess, and $12(75 \%)$ of 16 food isolates. Six $(75 \%)$ of eight raw chicken isolates, including four $(80 \%)$ of five from one major breeder, also had this profile. Moreover, all eight human and three food isolates linked to the large outbreak had this profile.

The second most common MLVA allele profile, 47-2-10-2-4-11, was exhibited by isolates recovered from mayonnaise samples taken eight months apart from a snack bar and the human isolate that was linked with food poisoning at this bar. Interestingly, this profile was also found in the only isolate from raw chicken marketed by another major breeder. An isolate from a parotid gland abscess also had this profile. The three other profiles were found mostly in isolates from blood cultures of patients with underlying diseases.

\section{Discussion}

A pandemic of $S$. Enteritidis was first noted in the late 1980's and has been attributed to infected poultry and contaminated eggs [13]. Salmonella serovar Enteritidis has been among the most prevalent serovars reported globally for several years. However, geographical variation in the isolation of $S$. Enteritidis has been noted. In 1995, $S$. Enteritidis was found to be the most frequently isolated serovar in about $88 \%$ of the countries in the European and American regions, but in none of the countries in the African region [14]. In 2000-2002, $S$. Enteritidis was again the most prevalent Salmonella serovar in humans globally and accounted for $85 \%$ of Salmonella cases in Europe, $38 \%$ in Asia and $26 \%$ in Africa [15], however, Mauritius was spared the pandemic until 2007. The reason for this is unclear but the fact that almost all chicken and eggs sold in Mauritius were locally produced before 2008, is probably a significant factor. However, since 2008, S. Enteritidis has been commonly isolated from clinical and food samples and, indeed, in 2008 and 2009, non-typhoidal Salmonella serogroup 0:9 displaced Salmonella serogroup $\mathrm{O}: 4$ as the most common serogroup recovered from human stools in Mauritius. There was also a simultaneous rise in the number of (nontyphoidal Salmonella) NTS isolates from blood cultures in 2008 and 2009, with Salmonella serogroup O:9 accounting for about $60 \%$ of the cases in these years. Isolation of any NTS from exudates had been infrequent in Mauritius until 2009 when almost threequarters of NTS isolates from exudates were $S$. Enteritidis. Thus, following the emergence of $S$. Enteritidis in the country, more invasive infections have been encountered than previously, particularly in patients with underlying diseases. Previously published studies have shown that $S$. Enteritidis are among a few serotypes that are associated with a higher risk of extra-intestinal infection [16,17]. Immunocompromised patients are also known to be at higher risk for the development of bacteremia with this serotype [17]. However, some of our patients, particularly those with abscesses, were not immunocompromised and were not known to be suffering from underlying diseases.

The main objective of the present study was to investigate the genetic relatedness among $S$. Enteritidis isolates causing gastrointestinal illness, bacteremia and other extraintestinal infections in patients from Mauritius and to potentially identify the source of transmission to humans. Given the limited discriminatory power of pulse-field gel electrophoresis (PFGE) to differentiate strains of Salmonella serovar Enteritidis, a more discriminatory molecular subtyping method (MLVA) was utilized [18]. Retrospective data analysis identified a marked increase beginning in 2008, in both human NTS serogroup O:9 infections and the recovery of NTS serogroup 0:9 from raw chicken and ready to eat food products in Mauritius. Although most isolates were not serotyped, the great majority were likely to have been Salmonella serovar Enteritidis as almost all of Salmonella serogroup O:9 isolates chosen for further studies were subsequently confirmed as this serotype.

Molecular and phenotypic subtyping of 47 representative isolates of $S$. Enteritidis revealed the presence of highly related strains in both humans and chicken, indicating that poultry is likely the main reservoir for human infections in Mauritius. Indeed, outbreaks caused by this serotype were associated 
mostly with chicken or egg-based products as the vehicle although infected foodhandlers may have been involved in a few cases. In some cases crosscontamination with chicken or eggs in the kitchen may have led to contamination of other types of food such as chili sauce.

All eleven stool and food isolates linked with the large outbreak associated with chicken tikka or kebab were indistinguishable by MLVA typing. Similarly, with one exception, all isolates linked to an outbreak had indistinguishable profiles, suggesting that the same strain was present in both the food and the clinical samples. In the case of the outbreak associated with the snack bar, one raw chicken isolate had a slightly different MLVA allele profile from the stool and mayonnaise isolates, possibly because isolation of $S$. Enteritidis in that particular chicken specimen was an incidental finding unrelated to contamination of the mayonnaise which was the probable vehicle in the outbreak. Even though only slight differences were identified by MLVA among the 47 isolates, typing data generally supported epidemiological information available on isolates that were considered to be related.

The MLVA typing results suggest that all isolates are of a single clone which is most likely to have arisen from a single source. However, further analysis of the diversity in strains circulating in Mauritius would be necessary to confirm this. Although, Salmonella serovar Enteritidis is known for not being particularly resistant to antimicrobials, the lack of even a single resistance determinant also supports the hypothesis of a very clonal distribution. It is unclear how this clone was introduced in the country but according to some veterinarians, imported contaminated fishmeal for poultry is suspected as a possible source.

Stool isolates were highly related or indistinguishable from isolates recovered from blood and exudate. These results suggest that the same clone is responsible for both intestinal and extra-intestinal infections. The emergence and spread of this organism has caused considerable morbidity in the country. The financial impact is also significant as extra-intestinal infections typically result in many days of work lost and increased cost of hospitalization.

The presence of clonally related strains and the limited number of poultry breeders in Mauritius offer clear opportunities for epidemiologic interventions. Control of Salmonella in broilers from the affected poultry producer could lead to a marked reduction in the number of cases of salmonellosis in humans.
Control strategies could include the use of Salmonellafree parent flocks and Salmonella-free chicken feeds, regular cleaning and disinfection of poultry houses, with empty resting periods between flocks, measures to prevent domestic and wild animals, including wild birds, from gaining access to the premises, vaccination of breeder and layer flocks [19,20], and intensive flock-level testing with destruction of infected flocks [21]. However, apparently expensive measures could eventually be cost-effective as reduction of Salmonella in food animals would most likely lower the burden of human infections and reduce the cost associated with such illnesses.

\section{Conclusions}

Epidemiologic studies indicate that the burden of disease due to $S$. Enteritidis increased dramatically in 2008 and 2009. Characterization of clinical and food isolates revealed that a highly clonal strain of $S$. Enteritidis is circulating among the human population and it can also be found in food specimens originating from a major poultry breeder. The homology between stool and extra-intestinal isolates suggests that one strain is causing both gastrointestinal and invasive infections. Identification of a common strain circulating among the human population and poultry establishments offers a clear opportunity for intervention. Controlling $S$. Enteritidis in the poultry population can have a direct impact on public health in Mauritius.

\section{Acknowledgements}

This work was supported by the World Health Organization Global Foodborne Infections Network (WHO GFN) (www.who.int/gfn). We would also like to thank Mrs. P Lan Keng Lun, Mr RK Lutchun, Miss N Kanaksabee, and all the technical staff of the bacteriology section of the CHL for their technical assistance. Additionally, we would like to thank Ms. Ashley Sabol from CDC for performing MLVA.

\section{References}

1. Todd EC (1997) Epidemiology of foodborne diseases: a worldwide review. World Health Stat Q 50: 30-50.

2. Hohmann EL (2001) Nontyphoidal salmonellosis. Clin Infect Dis 32: 263-269.

3. Swaminathan B, Gerner-Smidt P, Barrett T (2006) Focus on Salmonella. Foodborne Pathog Dis 3: 154-156.

4. Moussa MF (2007) Etude comparative des germes intestinaux isolés au laboratoire de référence de l'île Maurice entre 199899 et 2004-05. Diplôme Universitaire de santé publique à l'île Maurice. Université Victor Ségalen, Bordeaux 2 et Institut de Santé de Maurice. 
5. Ministry of Health \& Quality of Life (2011) Positivity rates of pathological tests carried out in public laboratories on certain selected pathogenic conditions 2008 - 2010. In Island of Mauritius Health Statistics Annual 2010. Available http://www.gov.mu/portal/goc/moh/file/statsm10/oth10m/Pat hoPositiveRates\%202008-2010.pdf Accessed 16 April 2013

6. Hendriksen RS, Vieira AR, Karlsmose S, Lo Fo Wong DM, Jensen AB, Wegener HC, Aarestrup FM (2011) Global monitoring of Salmonella serovar distribution from the World Health Organization Global Foodborne Infections Network Country Data Bank: results of quality assured laboratories from 2001 to 2007. Foodborne Pathog Dis 8:8 87-900.

7. Freeman R (1989) Bacteriology of normally sterile body fluids. In Hawkey P, Lewis D, editors. Medical Bacteriology, 1st edition. New York: Oxford University Press. 21-42.

8. Issack MI, Hendriksen RS, Lun PL, Lutchun RK, Aarestrup FM (2009) Salmonella enterica serovar Typhimurium in Mauritius linked to consumption of marlin mousse. Foodborne Pathog Dis 6: 739-41.

9. Pedler S, Graham G (2004) Bacteriology of intestinal disease. In Hawkey P, Lewis D editors. Medical Bacteriology, 2nd edition. New York: Oxford University Press. 177-213.

10. Grimont PA D and Weill FX (2007) Antigenic formulae of the Salmonella serovars, 9th edition. WHO Collaborating Center for Reference and Research on Salmonella, Institut Pasteur, Paris, France.

11. Agron PG, Walker RL, Kinde H, Sawyer SJ, Hayes DC, Wollard J, Andersen GL (2001) Identification by subtractive hybridization of sequences specific for Salmonella enterica serovar enteritidis. Appl Environ Microbiol 67: 4984-4991.

12. Hendriksen RS, Le Hello S, Bortolaia V, Pulsrikarn C, Nielsen EM, Pornruangmong S, Chaichana P, Svendsen CA, Weill FX, Aarestrup FM (2012) Characterization of isolates of Salmonella enterica serovar Stanley, a serovar endemic to Asia and associated with travel. J Clin Microbiol 50: 709-720.

13. Rodrigue DC, Tauxe RV, Rowe B (1990) International increase in Salmonella enteritidis: a new pandemic? Epidemiol Infec 105: 21-27.

14. Herikstad H, Motarjemi Y, Tauxe RV (2002) Salmonella surveillance: a global survey of public health serotyping. Epidemiol Infect 129: 1-8.

15. Galanis E, Lo Fo Wong DM, Patrick ME, Binsztein N, Cieslik A, Chalermchikit T, Aidara-Kane A, Ellis A, Angulo FJ, Wegener HC (2006) World Health Organization Global
Salm-Surv. Web-based surveillance and global Salmonella distribution, 2000-2002. Emerg Infect Dis 12: 381-388.

16. Jones TF, Ingram LA, Cieslak PR, Vugia DJ, Tobin-D'Angelo M, Hurd S, Medus C, Cronquist A, Angulo FJ (2008) Salmonellosis outcomes differ substantially by serotype. J Infect Dis 198: 109-114.

17. Dhanoa A, Fatt QK (2009) Non-typhoidal Salmonella bacteraemia: epidemiology, clinical characteristics and its association with severe immunosuppression. Ann Clin Microbiol Antimicrob 8: 15.

18. Boxrud D, Pederson-Gulrud K, Wotton J, Medus C, Lyszkowicz E, Besser J, Bartkus JM (2007) Comparison of multiple-locus variable-number tandem repeat analysis, pulsed-field gel electrophoresis, and phage typing for subtype analysis of Salmonella enterica serotype Enteritidis. J Clin Microbiol 45: 536-543.

19. Toyota-Hanatani Y, Ekawa T, Ohta H, Igimi S, Hara-Kudo Y, Sasai K, Baba E (2009) Public health assessment of Salmonella enterica serovar enteritidis inactivated-vaccine treatment in layer flocks. Appl Environ Microbiol 75: 100510010 .

20. Collard JM, Bertrand S, Dierick K, Godard C, Wildemauwe C, Vermeersch K, Duculot J, Van Immerseel F, Pasmans F, Imberechts H, Quinet C (2008) Drastic decrease of Salmonella Enteritidis isolated from humans in Belgium in 2005, shift in phage types and influence on foodborne outbreaks. Epidemiol Infect 136: 771-781.

21. FAO/WHO Global Forum of Food Safety Regulators, Marrakech, Morocco, 28 - 30 January 2002. Available: http://www.fao.org/DOCREP/MEETING/004/AB456E.HTM. Accessed November 2013

\section{Corresponding author}

Dr. Mohammad I. Issack

Central Health Laboratory, Victoria Hospital, Candos, Mauritius

Phone: +230 4270531

Fax: + 2304245848

E-mail: moissack@yahoo.com

Conflict of interests: No conflict of interests is declared. 